09

\title{
Влияние конструкции резонатора на ширину линии одномодовых вертикально-излучающих лазеров ближнего ИК-диапазона
}

\author{
(C) С.А. Блохин ${ }^{1}$, М.А. Бобров ${ }^{1}$, А.Г. Кузьменков ${ }^{1,2}$, А.А. Блохин ${ }^{1,2}$, \\ А.П. Васильев ${ }^{1,2}$, Ю.А. Гусева ${ }^{1,2}$, М.М. Кулагина ${ }^{1}$, \\ Ю.М. Задиранов ${ }^{1}$, Н.А. Малеев ${ }^{1,3}$, И.И. Новиков ${ }^{4}$, \\ Л.Я. Карачинский ${ }^{1,4}$, Н.Н. Леденцов ${ }^{5}$, В.М. Устинов ${ }^{1,2,6}$ \\ ${ }^{1}$ Физико-технический институт им А.Ф. Иоффе РАН, Санкт-Петербург, \\ Россия \\ ${ }^{2}$ Научно-технологический центр микроэлектроники и субмикронных \\ гетероструктур РАН, Санкт-Петербург, Россия \\ ${ }^{3}$ Санкт-Петербургский государственный электротехнический университет \\ „ЛЭТИ“, Санкт-Петербург, Россия \\ ${ }^{4} \mathrm{OOO} \mathrm{„Коннектор} \mathrm{Оптикс“,} \mathrm{Санкт-Петербург,} \mathrm{Россия}$ \\ ${ }^{5}$ VI Systems GmbH, Berlin, Germany \\ ${ }^{6}$ Санкт-Петербургский политехнический университет Петра Великого, \\ Санкт-Петербург, Россия \\ E-mail: blokh@mail.ioffe.ru
}

\section{Поступило в Редакцию 6 сентября 2017 г.}

Проведены исследования ширины линии излучения одномодовых вертикально-излучающих лазеров ближнего ИК-диапазона с активной областью на основе квантовых ям InGaAs/AlGaAs и различной конструкцией оптического микрорезонатора. При малых потерях на вывод излучения лазеры с $1 \lambda$-резонатором и инжекцией носителей через распределенные брэгговские отражатели демонстрируют ширину линии излучения $70 \mathrm{MHz}$ c ее ростом до $110 \mathrm{MHz}$ при повышении потерь на вывод излучения (соответствующая дифференциальная эффективность $\sim 0.65 \mathrm{~W} / \mathrm{A})$. Применение конструкции оптического резонатора с инжекцией носителей через внутрирезонаторные контакты и низкодобротные композиционные брэгговские решетки позволяет снизить ширину линии излучения до $40 \mathrm{MHz}$, несмотря на высокие потери на вывод излучения (соответствующая дифференциальная эффективность $\sim 0.6 \mathrm{~W} / \mathrm{A}$ ).

DOI: 10.21883/PJTF.2018.01.45432.17029 
Вертикально-излучающие лазеры (ВИЛ) ближнего ИК-диапазона $850 \mathrm{~nm}$ находят широкое применение в высокоскоростных оптических системах передачи данных, различных датчиках перемещения, лазерных интерферометрах и газовых сенсорах [1]. В последнее время большое внимание уделяется возможности использования одномодовых ВИЛ для задач оптической спектроскопии. Для классической геометрии вертикального оптического микрорезонатора ширина линии лазерной генерации ВИЛ с токовой апертурой, сформированной методом ионной имплантации (так называемая имплантационная токовая апертура), типично лежит в диапазоне $100-150 \mathrm{MHz}$ [2]. Однако в работах [3,4] была продемонстрирована возможность уменьшения ширины линии излучения ВИЛ спектрального диапазона $980 \mathrm{~nm} \mathrm{c}$ имплантационной токовой апертурой до $\sim 20-30 \mathrm{MHz}$, по-видимому за счет снижения потерь на вывод излучения (соответствует дифференциальной эффективности $\sim 0.1 \mathrm{~W} / \mathrm{A})$. Однако в ВИЛ с имплантационной токовой апертурой крайне сложно реализовать стабильный одномодовый режим генерации с малыми рабочими токами и высокой выходной мощностью из-за отсутствия эффективного оптического ограничения в латеральном направлении и высокого уровня потерь на радиационных дефектах, вызванных ионной имплантацией. Частичное решение указанной проблемы возможно для вариантов конструкции ВИЛ с селективным латеральным травлением слоев выводного зеркала вблизи резонатора (так называемая воздушная токовая апертура). Снижение уровня потерь на вывод излучения (дифференциальная эффективность $\sim 0.29 \mathrm{~W} / \mathrm{A}$ ) в ВИЛ спектрального диапазона $760 \mathrm{~nm}$ с воздушной токовой апертурой позволило достичь рекордно низкой ширины линии излучения $\sim 3 \mathrm{MHz}$ при выходной мощности $\sim 0.8 \mathrm{~mW}[5]$. Однако проблемы безызлучательной поверхностной рекомбинации и низкой механической надежности делают лазеры данного типа малоперспективными для практического применения.

В то же время ВИЛ, в которых токовое и оптическое ограничение реализовано методом селективного окисления слоев AlGaAs (так называемая оксидная токовая апертура) или с помощью формирования туннельного перехода (так называемая туннельная токовая апертура), позволяют преодолеть проблемы, характерные для ВИЛ с имплантационной токовой апертурой. Уменьшение потерь на вывод излучения (дифференциальная эффективность $\sim 0.12 \mathrm{~W} / \mathrm{A})$ позволило снизить ширину линии излучения ВИЛ спектрального диапазона $1550 \mathrm{~nm}$ с тунельной токовой апертурой до $\sim 20 \mathrm{MHz}$ при выходной мощности $\sim 0.8 \mathrm{~mW}[6]$.

Письма в ЖТФ, 2018, том 44, вып. 1 
Применение аналогичного подхода для ВИЛ спектрального диапазона $850 \mathrm{~nm}$ с оксидной токовой апертурой позволило уменьшить ширину линии изулчения до $\sim 50 \mathrm{MHz}$ при выходной мощности $0.7 \mathrm{~mW}$ [7]. Следует отметить, что снижение потерь на вывод излучения ведет к падению дифференциальной эффективности лазеров. Возможность дальнейшего заужения линии излучения ВИЛ связана с увеличением эффективной длины микрорезонатора, однако при этом необходимо сохранить низкий уровень внутренних оптических потерь. Прямое увеличение геометрической длины микрорезонатора сопряжено с проблемой обеспечения эффективной инжекции носителей в активную область (особенно дырок). В работе [8] было показано, что увеличение толщины одного слоя в легированном распределенном брэгговском отражателе (РБО) n-типа вблизи оптического резонатора до $\sim 1 \mu \mathrm{m}$ позволяет уменьшить ширину линии излучения ВИЛ спектрального диапазона $850 \mathrm{~nm}$ до $23 \mathrm{MHz}$ (при выходной мощности $\sim 0.45 \mathrm{~mW}$ ) и сохранить относительно низкий пороговый ток при дифференциальной эффективности $\sim 0.4 \mathrm{~W} / \mathrm{A}$. Однако такой подход сопряжен с падением модального усиления активной области из-за снижения фактора оптического ограничения в продольном направлении. В связи с этим представляется актуальным поиск альтернативных подходов сужения линии генерации ВИЛ при сохранении высокой эффективности прибора.

Настоящая работа посвящена исследованию ширины линии излучения одномодовых вертикально-излучающих лазеров ближнего ИК-диапазона с активной областью на основе квантовых ям InGaAs/AlGaAs и различной конструкцией оптического микрорезонатора: микрорезонатор с геометрическим расстоянием между РБО в одну длину волны (так называемый $1 \lambda$-резонатор) с инжекцией носителей через легированные зеркала (далее ВИЛ-1) и $1 \lambda$-резонатор с инжекцией носителей через внутрирезонаторные контакты и низкодобротные композиционные брэгговские решетки (далее ВИЛ-2). Рассмотрено влияние уровня потерь на вывод излучения при модификации выводного зеркала.

Конструктивно ВИЛ-1 представляет собой микрорезонатор с выводом излучения вверх и состоит из контактного слоя $n^{+}-\mathrm{GaAs}$, высокодобротного РБО $n$ - $\mathrm{Al}_{0.15} \mathrm{Ga}_{0.85} \mathrm{As} / \mathrm{Al}_{0.9} \mathrm{Ga}_{0.1} \mathrm{As}$, микрорезонатора $1 \lambda$-AlGaAs с активной областью на основе квантовых ям $\operatorname{In}_{0.08} \mathrm{Ga}_{0.92} \mathrm{As}$, высокодобротного РБО $p$ - $\mathrm{Al}_{0.15} \mathrm{Ga}_{0.85} \mathrm{As} / \mathrm{Al}_{0.9} \mathrm{Ga}_{0.1} \mathrm{As}$ с двумя апертурными слоями $p$ - $\mathrm{Al}_{0.98} \mathrm{Ga}_{0.02} \mathrm{As}$ и контактного слоя $p^{+}$-GaAs. Ocoбенности технологического процесса изготовления ВИЛ-1 приведены

Письма в ЖТФ, 2018, том 44, вып. 1 
в [9]. Вследствие анизотропии процесса селективного окисления слоев $\mathrm{AlGaAs}$ токовая оксидная апертура имеет форму квадрата [10].

Конструктивно ВИЛ-2 состоит из нижнего высокодобротного РБО $\mathrm{Al}_{0.15} \mathrm{Ga}_{0.85} \mathrm{As} / \mathrm{Al}_{0.9} \mathrm{Ga}_{0.1} \mathrm{As}$, внутрирезонаторного контактного слоя $n-\mathrm{Al}_{0.15} \mathrm{Ga}_{0.85} \mathrm{As}$, низкодобротной композиционной брэгговской решетки $n$ - $\mathrm{Al}_{0.15} \mathrm{Ga}_{0.85} \mathrm{As} / \mathrm{Al}_{0.9} \mathrm{Ga}_{0.1} \mathrm{As}$, микрорезонатора $1 \lambda$-AlGaAs с активной областью на основе квантовых ям $\operatorname{In}_{0.08} \mathrm{Ga}_{0.92} \mathrm{As}$, низкодобротной композиционной брэгговской решетки $p-\mathrm{Al}_{0.15} \mathrm{Ga}_{0.85} \mathrm{As}_{2} / \mathrm{Al}_{0.9} \mathrm{Ga}_{0.1} \mathrm{As}$ c одним апертурным слоем $p$-AlAs $/ \mathrm{Al}_{0.9} \mathrm{Ga}_{0.1} \mathrm{As}$, внутрирезонаторного контактного слоя $p-\mathrm{Al}_{0.15} \mathrm{Ga}_{0.85} \mathrm{As} / \mathrm{GaAs}$ и верхнего (выводного) диэлектрического $\mathrm{PБO} \mathrm{SiO}_{2} / \mathrm{Ta}_{2} \mathrm{O}_{5}$. Перед локальным осаждением диэлектрического РБО слой $p$-GaAs, обеспечивающий формирование омического контакта к внутрирезонаторному контактному слою р-типа, селективно удаляется в пределах светоизлучающей области лазера для предотвращения роста внутренних оптических потерь. Выбор системы материалов для диэлектрического РБО связан с необходимостью минимизации шероховатости интерфейсов и флуктуации показателя преломления аморфных слоев $\mathrm{SiO}_{2}$ и $\mathrm{Ta}_{2} \mathrm{O}_{5}$, изготовленных методом реактивного магнетронного распыления. Особенности технологического процесса изготовления ВИЛ-2 приведены в [11]. Конструкция апертурного слоя позволяет получить ромбовидную токовую оксидную апертуру и снимает вырождение фундаментальной моды по поляризации [12].

На рис. 1 представлены распределение интенсивности электромагнитного поля стоячей волны и профиль показателя преломления в микрорезонаторах ВИЛ-1 и ВИЛ-2. В случае ВИЛ-1 электромагнитное поле стоячей волны сконцентрировано в слое микрорезонатора $1 \lambda$-AlGaAs и экспоненциально затухает в зеркалах, при этом эффективная длина микрорезонатора с учетом конечной глубины проникновения электромагнитного поля в РБО составляет $\sim 1.5 \mu \mathrm{m}$. В то же время в ВИЛ-2 существенная доля электромагнитного поля стоячей волны оказывается сосредоточенной во внутрирезонаторных контактных слоях в силу малого коэффициента отражения композиционных брэгговских решеток. В результате электромагнитное поле стоячей волны в ВИЛ-2 распределено по большей длине структуры, чем в случае ВИЛ-1, что приводит к увеличению эффективной длины резонатора до $\sim 2.5 \mu \mathrm{m}$.

На рис. 2, $a$ приведены ватт-амперные характеристики одномодовых ВИЛ-1 и ВИЛ-2 с характерным размером токовой оксидной апертуры $2 \mu \mathrm{m}$, измеренные в непрерывном режиме работы при комнатной 

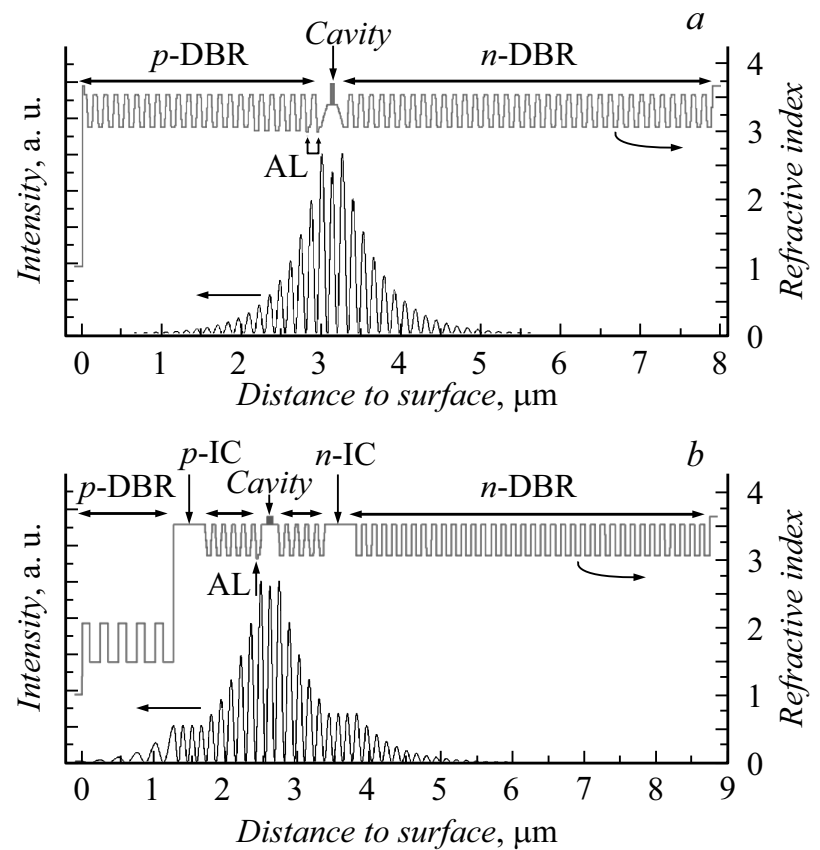

Рис. 1. Расчетное распределение электромагнитного поля стоячей волны и профиль показателя преломления в ВИЛ-1 (a) и ВИЛ-2 (b). DBR - распределенный брэгговский отражатель, cavity - резонатор, AL - апертурный слой, IC - внутрирезонаторные контактные слои $n$ - и $p$-типа.

температуре. Управление потерями на вывод излучения осуществлялось путем модификации коэффициента отражения выводного РБО с помощью сухого травления приповерхностного слоя верхнего (выводного) зеркала. При высоких потерях на вывод излучения ВИЛ-1 демонстрируют лазерную генерацию с пороговым током $\sim 0.56 \mathrm{~mA}$, дифференциальной эффективностью $\sim 0.65 \mathrm{~W} / \mathrm{A}$, максимальной выходной оптической мощностью $\sim 1.7 \mathrm{~mW}$, тогда как ВИЛ-2 демонстрируют лазерную генерацию с пороговым током $\sim 0.44 \mathrm{~mA}$, дифференциальной эффективностью $\sim 0.6 \mathrm{~W} / \mathrm{A}$, максимальной выходной оптической мощностью $\sim 2.2 \mathrm{~mW}$. Уменьшение потерь на вывод излучения ВИЛ-1 ведет к снижению порогового тока до $0.18 \mathrm{~mA}$, а также падению диф-

Письма в ЖТФ, 2018, том 44, вып. 1 

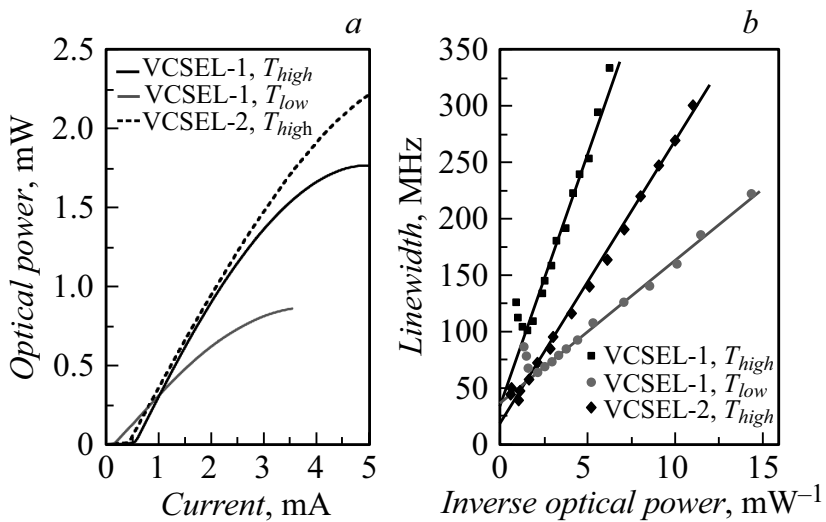

Рис. 2. Типичные ватт-амперные характеристики (a) и зависимости ширины линии излучения от обратной оптической мощности $(b)$ для ВИЛ-1 (VCSEL-1) и ВИЛ-2 (VCSEL-2) с характерным размером токовой оксидной апертуры $\sim 2 \mu \mathrm{m}$. Измерения проведены при температуре $20^{\circ} \mathrm{C}$. $T_{l o w}-$ низкие потери на вывод излучения, $T_{h i g h}$ - высокие потери на вывод излучения.

ференциальной эффективности до $0.36 \mathrm{~W} / \mathrm{A}$ и максимальной выходной оптической мощности до $0.9 \mathrm{~mW}$.

Поляризационные исследования ВИЛ-1 показали, что при высоких потерях на вывод излучения, несмотря на вырождение фудаментальной моды резонатора по поляризации, преобладает одно направление поляризации излучения с фактором подавления ортогональной поляризации (OPSR) более $15 \mathrm{~dB}$ во всем рабочем диапазоне токов, однако при малых потерях на вывод излучения наблюдаются переключение поляризации излучения и гистерезис. Такое поведение, по-видимому, обусловлено наличием слабого электрооптического эффекта или механических напряжений, которые ведут к снятию вырождения фундаментальной моды по поляризации, но с малой степенью дискриминации второй моды, что в условиях низких потерь на вывод излучения приводит к возможности достижения порогового условия обеими модами. В случае ВИЛ-2 фикисированная поляризация с OPSR более $15 \mathrm{~dB}$ наблюдается во всем рабочем диапазоне токов благодаря ромбовидной токовой оксидной апертуре [13].

На рис. 2, $b$ приведены результаты измерения ширины линии излучения доминирующей моды ВИЛ-1 и ВИЛ-2 с помощью сканиру-

Письма в ЖТФ, 2018, том 44, вып. 1 
ющего интерферометра Фабри-Перо Thorlabs SA-200 (разрешающая способность $7.5 \mathrm{MHz}$ ) в зависимости от обратной оптической мощности. Для обоих типов лазеров при превышении порога генерации с ростом тока накачки сначала наблюдается падение ширины линии излучения обратно пропорционально выходной оптической мощности согласно выражению $[1,14]$

$$
\Delta v_{L}=\frac{h v n_{s p} \eta_{0}}{4 \pi \tau_{p}^{2} P}\left(1+\alpha^{2}\right)+\Delta v_{0},
$$

где $P-$ выходная оптическая мощность, $h v-$ энергия излучаемых фотонов, $\tau_{p}$ - время жизни фотонов в резонаторе, $\eta_{0}-$ внешняя квантовая эффективность, $n_{s p}-$ фактор инверсной заселенности (типично $\sim 1.5$ ), $\alpha-$ фактор уширения спектральной линии (далее $\alpha$-фактор), $\Delta v_{0}$ - остаточная ширина линии, связанная с фликер-шумом или конкуренцией поперечных мод. Однако при дальнейшем росте тока накачки наблюдается сильнее отклонение от классической теории поведения ширины линии излучения в зависимости от выходной оптической мощности [14]: насыщение и уширение линии генерации одномодовых лазеров с ростом выходной оптической мощности. В результате при высоких потерях на вывод излучения ширина линии генерации ВИЛ-1 достигает минимального значения $\sim 100 \mathrm{MHz}$ при выходной оптической мощности $\sim 0.8 \mathrm{~mW}$ и затем увеличивается до $\sim 120-140 \mathrm{MHz}$ с ее ростом. Уменьшение потерь на вывод излучения ведет к двукратному росту времени жизни фотонов в резонаторе ВИЛ-1, однако ширина линии генерации ВИЛ-1 падает лишь до $65 \mathrm{MHz}$, а затем линия снова уширяется до $90 \mathrm{MHz}$ с ростом выходной оптической мощности, причем при фиксированном уровне выходной оптической мощности наблюдается слабое изменение ширины линии с модификацией времени жизни фотонов в резонаторе. Такое поведение, по-видимому, обусловлено увеличением $\alpha$-фактора вследствие более высокого значения вариации показателя преломления активной области с изменением концентрации носителей: с одной стороны, сдвиг резонансной длины волны вблизи порога, обусловленный изменением показателя преломления активной области, практически не зависит от величины порогового тока (т.е. от уровня потерь на вывод излучения), а с другой - концентрация носителей падает с уменьшением потерь на вывод. Полученные данные коррелируют с результатами для ВИЛ в классической геометрии микрорезонатора и суммарными потерями $\sim 0.4 \%$ на проход резонатоpa [8]. Дальнейшее уменьшение ширины линии ВИЛ-1 без увеличения

Письма в ЖТФ, 2018, том 44, вып. 1 
эфективной длины резонатора возможно лишь за счет еще большего снижения потерь на вывод излучения и минимизации внутренних оптических потерь $[4,6]$.

Благодаря увеличению эффективной длины резонатора ВИЛ-2 до $2.5 \mu \mathrm{m}$ удается получить более узкую линию генерации при высоком уровне потерь на вывод излучения по сравнению с ВИЛ-1 при идентичном уровне выходной оптической мощности. Так, ширина линии излучения ВИЛ-2 достигает минимального значения $\sim 40 \mathrm{MHz}$ при выходной оптической мощности $\sim 1.2 \mathrm{~mW}$. Следует отметить, что ширина линии излучения ВИЛ-2 не превышает уровня $50 \mathrm{MHz}$ вплоть до $2 \mathrm{~mW}$, когда наблюдается существенный тепловой загиб ваттамперной характеристики.

Причина неклассического поведения ширины линии излучения исследованных ВИЛ с ростом выходной оптической мощности, повидимому, связана с режимом работы одномодового лазера в условиях высокой плотности носителей и фотонов в микрорезонаторе, когда c ростом тока накачки утечка носителей и сильный саморазогрев ведут к быстрому насыщению усиления и падению дифференциального усиления и в конечном счете к росту $\alpha$-фактора $[15,16]$. Следует отметить, что уменьшение характерного размера токовой апертуры исследованных ВИЛ приводит к росту минимального значения ширины линии излучения. Данный факт связан с падением времени жизни фотонов в резонаторе и увеличением $\alpha$-фактора вследствие роста внутренних оптических потерь из-за рассеяния света на границе оксид-полупроводник и из-за дополнительного падения эффективности токовой инжекции ввиду роста утечки носителей при их повышенной концентрации. В связи с этим одновременное увеличение характерного размера апертуры ВИЛ-2 на 20-30\% и уменьшение потерь на вывод излучения ( $\sim 0.25 \%$ на проход) позволили снизить ширину линии генерации до уровня $\sim 20-25 \mathrm{MHz}$ при выходной оптической мощности $0.5-1 \mathrm{~mW}$ при дифференциальной эффективности $\sim 0.43 \mathrm{~W} / \mathrm{A}$. Возможные способы дальнейшего его сужения линии излучения ВИЛ-2 при сохранении высокой эффективности лазера связаны с оптимизацией конструкции композиционных решеток и увеличением толщины внутрирезонаторного контактного слоя $n$-типа.

Таким образом, проведенные исследования показали, что применение конструкции оптического микрорезонатора с инжекцией носителей через внутрирезонаторные контакты и низкодобротные композиционные брэгговские решетки позволяет сузить линию генерации ВИЛ

Письма в ЖТФ, 2018, том 44, вып. 1 
ближнего ИК-диапазона до 40-50 MHz при выходной оптической мощности 1-2 $\mathrm{mW}$ и дифференциальной эффективности $\sim 0.6 \mathrm{~W} / \mathrm{A}$. Полученные приборные характеристики демонстрируют перспективы использования ВИЛ данной конструкции в задачах оптической спектроскопии.

Работа выполнена при частичной поддержке программы Президиума РАН № 1 „Наноструктуры: физика, химия, биология, основы технологий“ (проект № 1144).

\section{Список литературы}

[1] Michalzik R. // VCSELs: Fundamentals, technology and applications of verticalcavity surface-emitting Lasers / Ed. R. Michalzik. Berlin: Springer-Verlag, 2013. P. 560.

[2] Kuksenkov D., Feld S., Wilmsen C. et al. // Appl. Phys. Lett. 1995. V. 66. Iss 3. P. 277.

[3] Reiner G., Zeeb E., Möller B. et al. // IEEE Photon. Technol. Lett. 1995. V. 7. Iss. 7. P. 730.

[4] Schmid W., Jung C., Weigl B. et al. // IEEE Photon. Technol. Lett. 1996. V. 8. Iss. 18. P. 1288.

[5] De Sopra F.M., Zappe H.P., Moser M. et al. // IEEE Photon. Technol. Lett. 1999. V. 11. Iss. 12. P. 1533.

[6] Bacou A., Rissons A., Mollier J.-C. // Proc. SPIE. 2008. V. 6908. P. 69080F.

[7] Serkland D.K., Peake G.M., Geib K.M. et al. // Proc. SPIE. 2006. V. 6132. P. 613208.

[8] Serkland D.K., Keeler G.A., Geib K.M., Peake G.M. // Proc. SPIE. 2009. V. 7229. P. 722907.

[9] Блохин С.А., Карачинский Л.Я., Новиков И.И. и др. // ФТП. 2014. Т. 48. B. 1. C. 81.

[10] Choquette K.D., Geib K.M., Ashby C.I.H. et al. // IEEE J. Selected. Topics Quantum Electron. 1997. V. 3. Iss. 3. P. 916.

[11] Малеев Н.А., Кузьменков А.Г., Кулагина М.М. и др. // ФТП. 2013. Т. 47. B. 7. C. 985.

[12] Бобров М.А., Малеев Н.А., Блохин С.А. и др. // ФТП. 2016. Т. 50. В. 10. C. 1408 .

[13] Nazaruk D.E., Blokhin S.A., Maleev N.A. et al. // J. Phys. Conf. Ser. 2014. V. 572. P. 012036.

[14] Henry C.H. // IEEE J. Quantum Electron. 1982. V. 18. Iss. 2. P. 259.

[15] Dowd P., Summers H.D., White I.H. et al. // Electron. Lett. 1995. V. 31. Iss. 7. P. 557.

[16] Halbritter H., Shau R., Riemenschneider F. et al. // Electron. Lett. 2004. V. 40. Iss. 20. P. 1266.

Письма в ЖТФ, 2018, том 44, вып. 1 